\title{
BacSim, a simulator for individual-based modelling of bacterial colony growth
}

\author{
Jan-Ulrich Kreft, ${ }^{1}$ Ginger Booth ${ }^{2}$ and Julian W. T. Wimpenny ${ }^{1}$ \\ Author for correspondence: Jan-Ulrich Kreft. Tel: +44 1222874000 ext. 6036. Fax: +44 1222874305. \\ e-mail: Kreft@,cardiff.ac.uk
}

1 School of Pure and Applied Biology, Cardiff University, PO Box 915, Cardiff CF1 3TL, UK

2 Center for Computational Ecology, Yale Institute for Biospheric Studies, PO Box 208104, New Haven, CT 06520-8104, USA
The generic, quantitative, spatially explicit, individual-based model BacSim was developed to simulate growth and behaviour of bacteria. The potential of this approach is in relating the properties of microscopic entities - cells - to the properties of macroscopic, complex systems such as biofilms. Here, the growth of a single Escherichia coli cell into a colony was studied. The object-oriented program BacSim is an extension of Gecko, an ecosystem dynamics model which uses the Swarm toolkit for multi-agent simulations. The model describes bacterial properties including substrate uptake, metabolism, maintenance, cell division and death at the individual cell level. With the aim of making the model easily applicable to various bacteria under different conditions, the model uses as few as eight readily obtainable parameters which can be randomly varied. For substrate diffusion, a two-dimensional diffusion lattice is used. For growth-rate-dependent cell size variation, a conceptual model of cell division proposed by Donachie was examined. A mechanistic version of the Donachie model led to unbalanced growth at higher growth rates, whereas including a minimum period between subsequent replication initiations ensured balanced growth only if this period was unphysiologically long. Only a descriptive version of the Donachie model predicted cell sizes correctly. For maintenance, the Herbert model (constant specific rate of biomass consumption) and for substrate uptake, the Michaelis-Menten or the Best equations were implemented. The simulator output faithfully reproduced all input parameters. Growth characteristics when maintenance and uptake rates were proportional to either cell mass or surface area are compared. The authors propose a new generic measure of growth synchrony to quantify the loss of synchrony due to random variation of cell parameters or spatial heterogeneity. Variation of the maximal uptake rate completely desynchronizes the simulated culture but variation of the volume-at-division does not. A new measure for spatial heterogeneity is introduced: the standard deviation of substrate concentrations as experienced by the cells. Spatial heterogeneity desynchronizes population growth by subdividing the population into parts synchronously growing at different rates. At a high enough spatial heterogeneity, the population appears to grow completely asynchronously.

Keywords: individual-based modelling, colony growth, growth synchrony, spatial heterogeneity

\section{INTRODUCTION}

The fundamental unit of bacterial life, encapsulating

Abbreviations: 2D, two-dimensional; IbM, individual-based model/ modelling. action, information storage and processing, as well as variability, is the cell. It therefore seems appropriate to construct ecological models in terms of individual cells and their behaviour. This paper introduces spatially explicit individual-based modelling ( $\mathrm{IbM}$ ) to microbial ecology. The great potential of $\mathrm{IbM}$ lies in addressing 


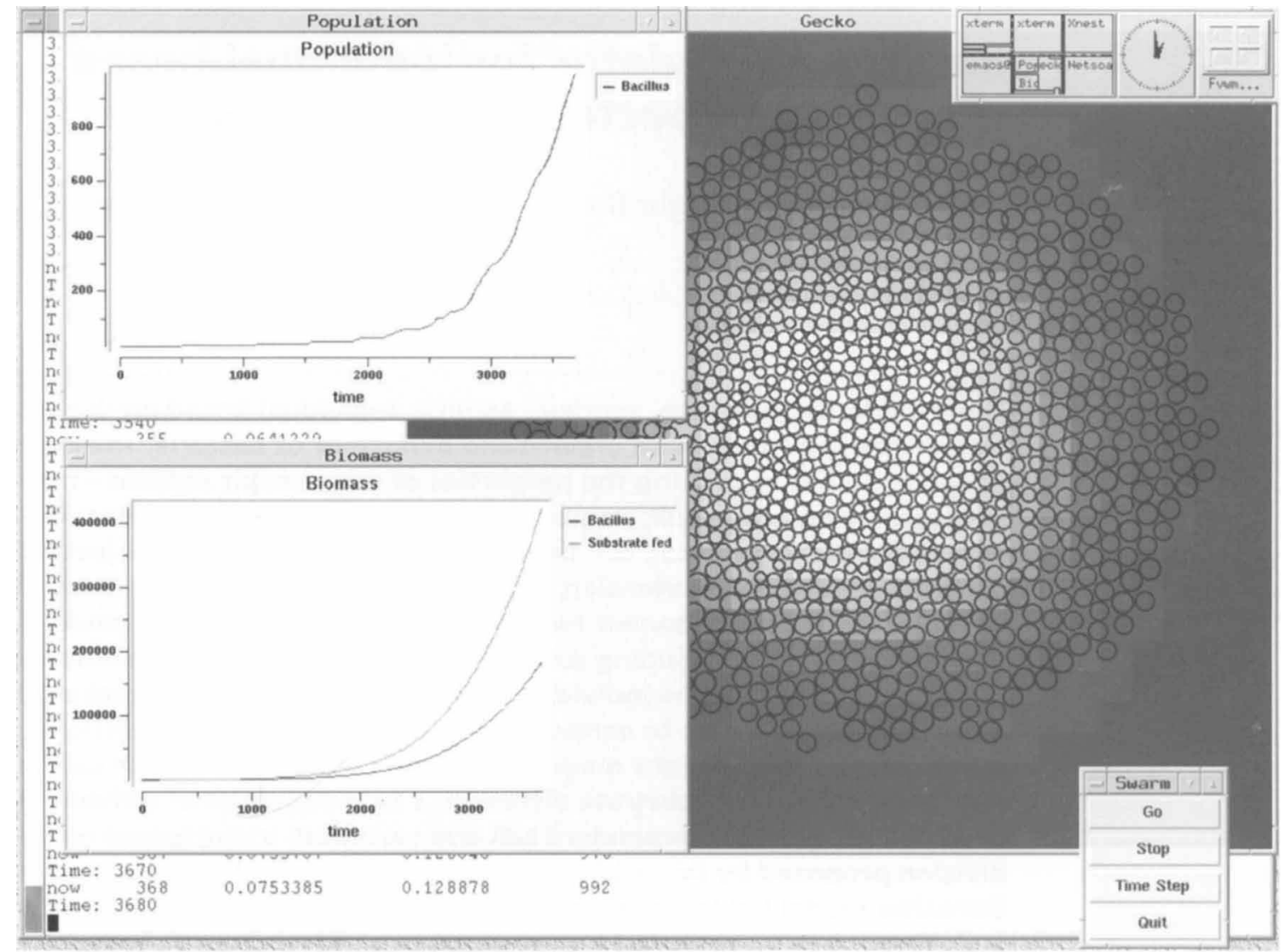

Fig. 1. Screen shot of a Gecko simulation at time $368 \mathrm{~min}$. The windows with the population growth curve (labelled Population; units: cell number and time-steps), the total substrate-consumed and total dry-mass curves (labelled Biomass; units: fg dry mass or substrate and time-steps), the window with the simulation control panel (labelled Swarm), the window displaying the landscape with the growing colony (labelled Gecko), and the background window showing the data dumped to one of the log files, can all be seen. In the Gecko window, bacterial cells are drawn as circles (the circle radius is that which a spherical cell of this volume would have). The substrate concentration gradient is visualized as follows: the darker the square, the higher the concentration (logarithmic scale).

the following question: is it possible to create a macroscopic world from data on microscopic entities?

The existing extensive literature on modelling bacterial colony growth is based on classical mathematical population models (Pirt, 1967; Grimson \& Barker, 1994) and cellular automaton models (Ben-Jacob et al., 1994; Shapiro \& Dworkin, 1997). The former have been used to describe growth of the colony as a unit and are limited to simple colony shapes. The latter have been used to explain qualitatively colonial pattern formation associated with different nutritional regimes. Picioreanu et al. (1998a, b) have used a combination of both approaches to model biofilm growth. IbM has so far not been used to model colony growth or more complex structures such as biofilms, which can be viewed as an assemblage of (micro)colonies. Such systems can be constructed to be more general, allowing one to treat biofilms, colonies and other microbial assemblages as special cases of a unified model. They can be based on the known physiology of individual cells and thus be quantitative. They provide the most complete frame- work for modelling whilst not suffering from many of the limitations inherent in the other approaches. On the other hand, such models require more microscopic data and have higher computational demands.

IbMs have been constructed in a variety of ways (DeAngelis \& Gross, 1992). They all have in common that individuals are treated either individually or grouped into cohorts of similar individuals, whereas population-level models do not allow for differences between individuals. One important characteristic of IbMs is that they do not specify any global (populationlevel) laws such as exponential population growth. If exponential growth of the population emerges, it does so as a result of the substrate uptake, metabolism and maintenance of each of the individual cells. Many IbMs also allow for spatial differences, which are so important in microbial ecology (Wimpenny, 1992). To facilitate future extensions and modifications, in this study we constructed a flexible model structure with objectoriented programming techniques.

Constructing a single-cell model as the basis for a 
population model meets with problems specific to microbiology, that is obtaining single-cell properties from population-average measurements which so far constitute the bulk of the microbiological literature (Davey \& Kell, 1996). Single bacterial cell models have been published for Escherichia coli (Domach et al., 1984; Joshi \& Palsson, 1988) and Bacillus subtilis (Jeong et al., 1990). The purpose of these studies was to integrate subcellular processes into whole-cell models, necessitating the use of much information on the biochemistry of these bacteria. Such information is incomplete or unavailable for other species and indeed may not be totally satisfactory for $E$. coli. The purpose of our model, in contrast, is to integrate cellular processes into a generic population model. It therefore implements a much simpler metabolism, treating the cell as a black box as far as possible, and requires as few as eight parameters that are generally available or easily measurable. The simplified $E$. coli model of Jaworska $e t$ al. (1996) uses 22 parameters and the B. subtilis model of Jeong et al. (1990) uses nearly 200. In common with these authors, we believe that fitting a model to empirical data has to be avoided if the purpose of the model is not descriptive but predictive. For us, prediction is more than specifying a list of model assumptions which generate macroscopic structures that appear to look realistic, e.g. colony morphology. The aim is to obtain falsifiable, that is quantitative, predictions from entering physiologically based assumptions and measurable parameters into the model.

Here, BacSim is described, and results on cell division, growth curves and loss of growth synchrony are presented. New measures for synchrony and spatial heterogeneity are introduced.

Movies of growing colonies and additional screen shots, as well as the source code of the simulator and an on-line document, can be accessed on our web page (http:// www.eeb.yale.edu/ginger/bacillus/bacsim.html).

\section{THE MODEL}

BacSim is an extended and modified version of Gecko. Gecko is an 'ecological flight simulator' which has been used to study ecosystem dynamics such as trophic interactions in food webs (Schmitz \& Booth, 1997; Booth, 1997). Gecko has been written in the object-oriented language Objective- $\mathrm{C}$ using the Swarm toolkit for multi-agent simulations under development at the Santa Fe Institute, New Mexico, USA (Minar et al., 1996). Swarm's objective is to provide a common simulation platform for agent-based modelling in economics, anthropology, geography, ecology, ethology, and other fields concerned with complex adaptive systems. It is a collection of software libraries which provides the functionality common to all such applications.

The principle of BacSim is to represent each simulated bacterial cell electronically as an object instance of an objectoriented program. These objects are called agents since they are independent entities with their own state (set of parameter values) and behaviour. Nevertheless, they are all of the same type ('class' in object-oriented terminology), having the same potential regarding state and behaviour.

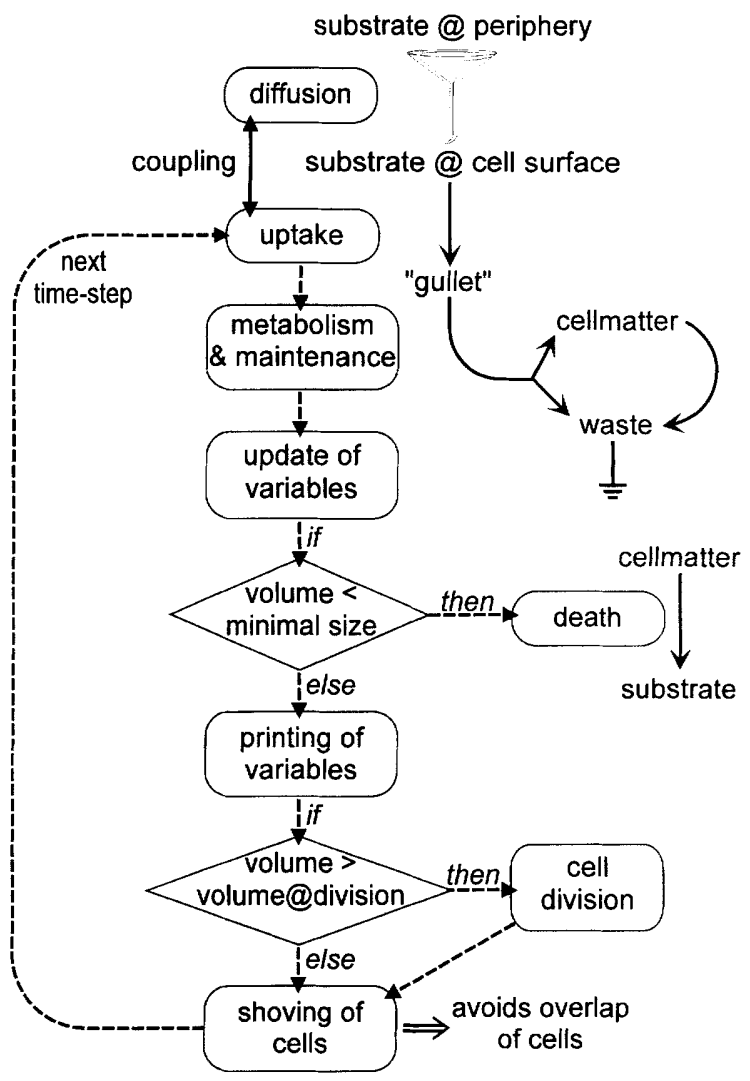

Fig. 2. Graphical pseudo-code of bacterial agent activities. Dashed arrows denote the sequence of methods (methods in boxes) carried out at each time-step by each agent. The rhomboid if-boxes denote decision methods. The funnel symbolizes diffusional substrate flux from source (the reservoir bordering on the diffusion lattice) to sink (the cells in the colony). Simple arrows indicate metabolic conversions. The waste produced from substrate (due to metabolism) and from cell matter breakdown (due to maintenance) is set to zero after conversions (earth symbol). The double arrow denotes the coupling of diffusion and uptake methods in the program. The shoving of cells avoids the temporary overlap of cells that would otherwise ensue.

BacSim agents exist in a continuous 2D space and have free range and extent - they occupy and compete for space. The agents are actually spheres, but they are projected onto this $2 \mathrm{D}$ space as circles (Fig. 1). For substrate diffusion, a lattice (Fig. 1) with variable grid granularity and overall size is used to simulate diffusion gradients quantitatively. Agents take up substrate from the lattice elements in which they are located. Time is discrete and agents repeat their schedule of activities once every time-step (Fig. 2). We used time-steps of $0 \cdot 1 \mathrm{~min}$. A compilation of the model parts and their implementation is given in Table 1.

Substrate diffusion. The diffusion lattice is initially filled with substrate at a given concentration. In all simulations reported here, a reservoir of constant substrate concentration that borders the diffusion lattice was also filled with the same substrate concentration. The substrate concentration of the colony periphery was always only marginally lower than the reservoir concentration which is stated in Results. For the coupled diffusion and uptake subroutines, a variable and self- 
adjusting number of substeps per time-step was employed for computational efficiency reasons. (Note that this substepping scheme is a mere technicality that does not bear on the global time-step of $0.1 \mathrm{~min}$ which drives time and sets the time precision of the model.) It is hard to overemphasize the importance of bacteria being small (Koch, 1996a). With regard to the model, it was necessary to make up to 10000 uptake steps min $^{-1}$ coupled to diffusion steps at intermediate substrate concentrations because otherwise the cells would have consumed more substrate per step than was available. In other words, the cells can clear their surroundings of substrate in about $0 \cdot 1 \mathrm{~ms}$. This way, diffusion limitations as artefacts of the model can be avoided. The diffusion limitations observed are therefore natural.

\section{Characteristics of bacteria agents}

Substrate uptake. Glucose is assumed to be the limiting substrate. Its uptake is considered to follow either the Michaelis-Menten equation or the Best equation (Koch, 1997). Uptake is also considered to be proportional either to dry mass or to surface area. Metabolism of the substrate taken up does not limit substrate uptake. Neither substrate thresholds nor exponential population growth are assumed.

Metabolism, maintenance and death. Metabolism converts substrate into biomass and waste at a fixed efficiency given by the yield constant. Maintenance reactions convert biomass into waste also. Waste is then discarded without effect. Maintenance is considered to be proportional to dry mass or to surface area. Maintenance under starvation conditions causes cells to shrink as biomass functions as an energy reserve. Cells die if their biomass falls below a minimum value. On death, biomass is converted back into substrate at a fixed efficiency of unity, thus allowing cryptic growth under starvation conditions. In fact, the shrinkage rate was much too low to lead to death during the time span used in our simulations. The metabolism implemented is as simple as possible. If desired, a more sophisticated metabolism could be implemented where information on the growth-rate-dependent variability of cellular composition or energetic efficiency is available.

Surface area. If used for maintenance or uptake, the surface area is calculated with equation (7). Note that apart from the surface area calculation for the modelling of uptake or maintenance, the cells are simulated as spheres which are represented as circles (for example see the screen shot in Fig. 1).

Cell division. The model of cell division from Donachie \& Robinson (1996) was implemented in three different ways: these are described in detail together with the results. The aim is to model the dependence of cell size on growth rate. The cell division process is completed instantaneously. It leads to two identical daughter cells in juxtaposition. No assumptions were made concerning growth synchrony.

Random variation of cell parameters. As each simulated cell has its own set of parameters which is an independent copy of the list of default parameter values, variation is straightforward. New values were obtained by random draws from a Gaussian distribution with a chosen coefficient of variation while discarding all values outside the $\pm 2 \sigma$ range as well as changes of sign (these precautions are necessary with distributions ranging from $-\infty$ to $+\infty$ ). As a starting value, we always used the default given in Table 2 , thereby avoiding unrestrained 'evolution'. If the current value for the maximal uptake rate was used as a starting point, those cells with higher rates would be more likely to produce offspring with even higher rates that would soon outgrow all other cells (data not shown). In reality, this tendency would be counteracted by diffusional and thermodynamic limitations and the metabolic costs of enzyme synthesis. Since modelling these constraints is beyond the scope of this paper, this unrealistic pseudoevolution was avoided by letting parameters deviate from the default. This decision is supported by the observation that variations in growth rate of individual colonies of a clonal culture are only temporary (Hughes, 1955).

Expansion of the growing colony. Agents shove each other to simulate the pressure generated by a growing colony in order to avoid overlap of cells. This makes it unnecessary to assume a maximum biomass or cell density in the colony as an upper limit. As data on the volume fraction of a wet colony occupied by cells are scarce, we adjusted the shoving so that cells merely did not overlap, resulting in a colony somewhat lighter than close-packed (see Fig. 6). Pirt (1967) reported a colony density of $E$. coli of $0 \cdot 1 \mathrm{~g}$ dry mass $\mathrm{ml}^{-1}$ that can be used to estimate an interstice of $77 \%$ assuming a water content of cells of $70 \%$ (Neidhardt \& Umbarger, 1996). Grimson \& Barker (1994) found the cellular volume fraction of a Salmonella typhimurium colony to be close to the typical value for closedpacked hard spheres of $\approx 64 \%$.

Running the model. The parameters we have used for the model are summarized in Table 2. The program (Gecko version 0.4 , Swarm library version 961002) was run on a Pentium133 PC with $64 \mathrm{MB}$ RAM under Linux as operating system (see the Swarm web page http://www.santafe.edu/ projects/swarm/ for a list of required software). Data output from the model was extracted and transformed as required with standard Unix tools (uniq, gawk). All statistical analyses and non-linear fits were performed with Dataplot (http://www.itl.nist.gov/div898/software/dataplot.html/ homepage.htm).

\section{RESULTS AND DISCUSSION}

\section{Model validation}

To facilitate a direct input of literature parameters the model reads in a file of parameters that can be entirely calculated from published figures (Table 2). Fitting the input parameters to obtain the desired output was unnecessary and was avoided. As a control of numerical correctness and program design, we verified that the model output agreed with the model input within the limits of numerical precision of both the simulation and the subsequent analysis of output data (Table 2). For the discrete lattice approximation of diffusion, a calibration factor had to be determined once by a computer simulation of a standard experiment to determine the diffusion coefficient (Adam et al., 1977). To do this, the diffusion lattice was initialized with unit substrate concentration in the left half and zero substrate concentration in the right half. Then, diffusion was allowed to proceed for a known number of time-steps as long as the left- and rightmost lattice parts still retained the original substrate concentration. The maximum of the 
Table 1. Equations and algorithms used in the model

Parameters are given in Table 2.

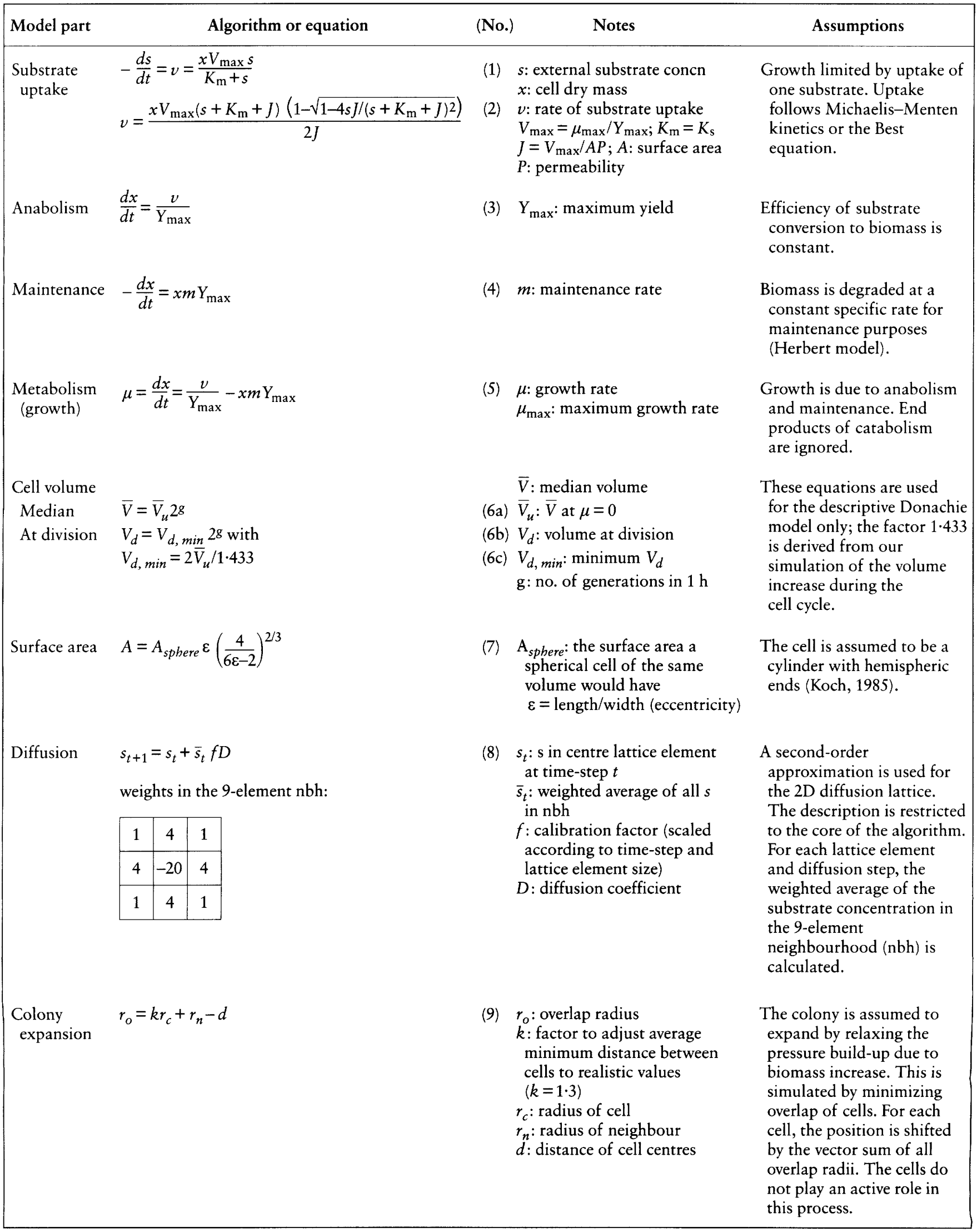


Table 2. Parameters used as input in model construction and the resulting model output

We have chosen reliable values typical for $E$. coli. If uptake is to be described by the Best equation, three instead of two parameters are needed for uptake. Their values, corresponding to the same data set, can be found in Koch \& Wang (1982). For surface area calculations, an average eccentricity of the cells of 4.4 (Donachie $\&$ Robinson, 1996) was used.

\begin{tabular}{|c|c|c|c|c|}
\hline Parameter & Units & Input & Model output \pm SD & Reference \\
\hline $\begin{array}{l}\mu_{\max }: \text { maximum growth rate } \\
K_{\mathrm{s}}: \text { half-saturation constant }\end{array}$ & $\min ^{-1}$ & $\begin{array}{c}0.0205 \\
2 \cdot 34 \times 10^{-3}\end{array}$ & $\begin{array}{c}0 \cdot 020428 \pm 1 \cdot 7 \times 10^{-8} \\
2.3328 \times 10^{-3} \pm 8.2 \times 10^{-9}\end{array}$ & $\begin{array}{l}\text { Koch \& Wang (1982), batch growth } \\
\text { Koch \& Wang (1982), batch growth }\end{array}$ \\
\hline $\begin{array}{l}Y_{\max }: \text { apparent yield at } \mu_{\max } \\
\text { corrected for maintenance }\end{array}$ & $\frac{\text { fg dry mass }}{\text { fg glucose }}$ & $0 \cdot 4444$ & $0 \cdot 4437 \pm 8 \cdot 0 \times 10^{-5}$ & Neijssel et al. (1996) \\
\hline $\begin{array}{l}\bar{V}_{u}: \text { median cell volume at } \mu=0 \\
\text { Time for replication }+ \text { cell } \\
\text { division (only mechanistic } \\
\text { Donachie models) }\end{array}$ & $\begin{array}{c}f l \\
\min \end{array}$ & $\begin{array}{l}0 \cdot 4 \\
60\end{array}$ & $\begin{array}{c}0 \cdot 396 \pm 1.8 \times 10^{-4} \\
\text { Not applicable }\end{array}$ & $\begin{array}{l}\text { Donachie \& Robinson (1996) } \\
\text { Donachie \& Robinson (1996) }\end{array}$ \\
\hline Cell density (dry mass) & $\mathrm{fg} \mathrm{fl}^{-1}$ & 290 & Not applicable & Shuler et al. (1979) \\
\hline $\begin{array}{l}D \text { : diffusion coefficient for } \\
\text { glucose }\end{array}$ & $\mu \mathrm{m}^{2} \min ^{-1}$ & 40680 & Not applicable & Adam et al. (1977) \\
\hline
\end{tabular}

concentration gradient was read from the middle horizontal row of the lattice to calculate the diffusion coefficient resulting from a known input parameter. From this ratio, a calibration factor was established for simple conversion of a literature diffusion coefficient into the model input value.

\section{Uptake}

As substrate has to be taken up through the cell's surface, it is straightforward to assume that uptake is proportional to surface area (Kooijman et al., 1991; Button, 1993). The changes of surface-to-volume ratio during the cell cycle would result in non-exponentiality of the cell's growth curve if uptake limits growth. However, after a long controversy, it is now generally assumed that the growth of a single cell does not deviate significantly from the exponential growth law (Koch, 1993). Two considerations might explain this. Firstly, the surface-to-volume ratio might change only slightly during the cell cycle since cell width changes during the cell cycle result in maintaining a more constant ratio (Domach et al., 1984). Secondly, most substrates are transported into the cell by proteins, which might be incorporated into the membrane at the same rate as the increase of cytoplasm and synthesis of cytoplasmic protein. Then, uptake and metabolic potential would increase proportionately despite changes of the surfaceto-volume ratio during the cell cycle. If transporter incorporation into the membrane is indeed proportional to overall protein synthesis, uptake and catabolic activities of the cell are kept in balance during the cell cycle, sparing the cell additional regulatory steps.

To illustrate the magnitude of the deviation from nonexponentiality due to surface area dependent uptake, we compared simulated growth with uptake proportional to either surface area or volume. The deviation of the growth curve from an exponential fit curve is hardly visible in the primary plot, but the residuals curve (residuals $=$ observed - predicted) clearly shows faster than exponential growth at the beginning of the cell cycle and slower growth at the end in the case of surfacedependent uptake (Fig. 3). Maintenance was not surface dependent in either case. The overall growth rate of the cell during one cycle is equal to the population growth rate in both cases. The autocorrelation coefficient of the relative residuals quantifies the noisiness of the residuals curve, with a value of 0.98 for perfect exponential growth (Fig. 3a) and -0.009 for surface-dependent growth (Fig. 3b). The extent of the deviation from exponentiality can be given by the range of the relative residuals, with a value of $3 \times 10^{-6}$ for Fig. 3(a) and $2 \times 10^{-2}$ for Fig. 3 (b).

In the absence of evidence for surface area proportionality of growth and the effort required to model the increase of surface area during the cell cycle adequately [cell width, length and eccentricity all depend on growth rate and change during the cell cycle; also, they differ from strain to strain (Donachie \& Robinson, 

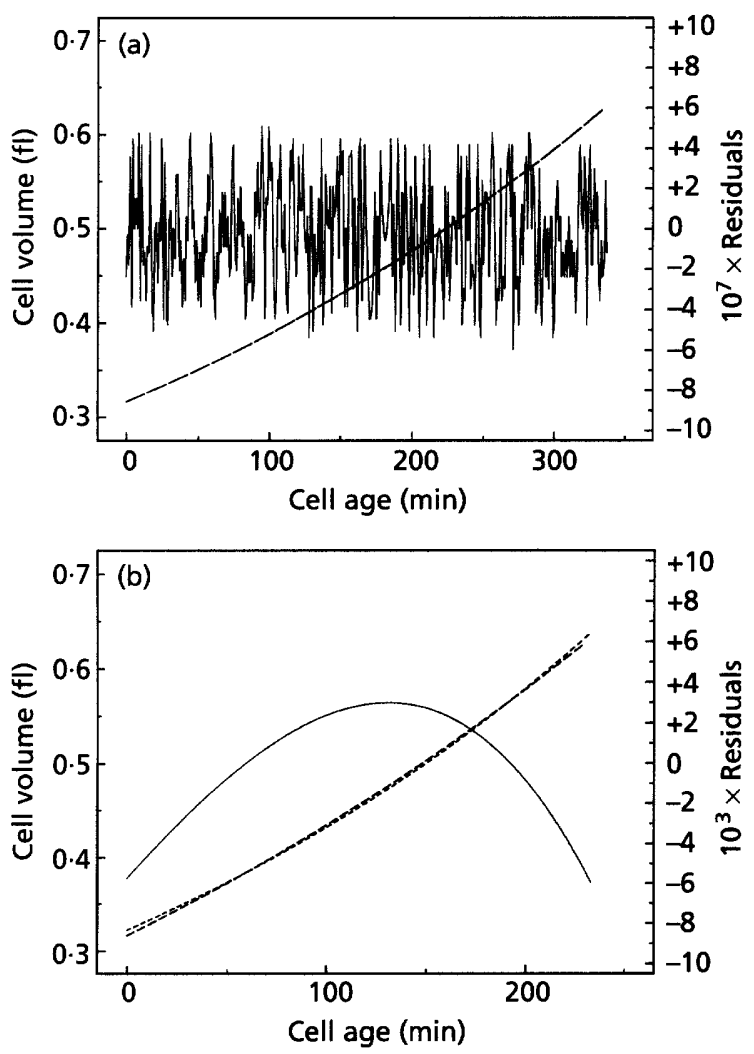

Fig. 3. Deviations from exponential cell growth due to surfacearea-dependent uptake. ---, Simulated growth curve; ---, fitted curve; —-, residuals (simulated data minus fitted curve). (a) Perfect exponential growth in the case of volumedependent uptake. The residuals curve shows noise, but no systematic deviation from exponentiality. (b) Non-exponential growth in the case of surface-area-dependent uptake. The residuals curve shows systematic deviation from exponentiality (noise not visible at this scale). Growth is faster than exponential at the beginning of the cell cycle, but slower than exponential at the end.

1996)], it appears best to use dry mass proportionality throughout. Also, this approach makes the model applicable to different cell shapes. Note that however the growth of a single cell is modelled, the asynchronous growth of a population of cells is exponential.

\section{Maintenance}

The simplest models for maintenance with a constant demand per biomass are those of Pirt (1965) and Herbert (1958), which differ only in assuming different sources for the maintenance energy, substrate or biomass, respectively. We have used the formulation by Herbert because his model behaves well in the case of low substrate concentrations where maintenance is especially important. In the Herbert model, no substrate is consumed at zero substrate concentration and the cells are allowed to shrink, in contrast to the Pirt model, where growth is always positive. Due to the postulated substrate concentration independence of maintenance, the Pirt model even describes consumption of substrate in its absence.

Maintenance energy is a collective term for all energy expenditures not directly contributing to growth. It thus encompasses energy costs for different purposes and subject to different regulation (Tempest \& Neijssel, 1984). For a more mechanistic understanding and modelling of the cell's energetics, it might be helpful to distinguish maintenance requirements proper from losses due to futile cycles, etc. Since most of this maintenance energy proper is spent on providing solute gradients across the cell membrane (Tempest $\&$ Neijssel, 1984), the maintenance rate can be considered to be surface area dependent.

Surface area dependence of maintenance, as with surface-dependent uptake, causes deviation from exponentiality of the cell's growth curve, but to a lesser degree (one order of magnitude) and in opposite directions (data not shown). The extent of deviation, given by the range of relative residuals, is $3 \times 10^{-3}$ and the autocorrelation coefficient of 0.99 signifies absence of noise in the residuals curve.

\section{Cell division}

Correctly simulating cell size as a function of growth rate is an important requirement for models in microbial ecology since the cell size affects starvation survival and metabolic rates. Donachie (1968) found, for cells growing at all growth rates, that cell masses at which initiation of DNA replication is triggered are multiples of one particular mass. Donachie concluded that cell division occurs at a fixed time $(60 \mathrm{~min}$ for E. coli, consisting of the time for DNA replication plus that for cell division) after the cell has reached the trigger volume or multiples thereof. This model is attractive due to its simplicity.

We have implemented this model, which we will call the mechanistic Donachie model, in our code. In addition, we implemented an extension of this model, called the mechanistic Donachie model with eclipse period. The eclipse period is the minimal interval between divisions and is implemented simply as an additional requirement to be met before cell division is allowed. This extension was made in order to improve the performance of the mechanistic Donachie model as it did not result in balanced growth at higher growth rates (Fig. 4). Although such an eclipse period has been described, the empirical value of about $12 \mathrm{~min}$ (Helmstetter, 1996) is much lower than the value which was required to force the mechanistic Donachie model to produce balanced growth, which was equal to the minimum doubling time.

Donachie also proposed equation (6a) describing the dependence of the median cell volume on growth rate. We have also implemented this model, which we call the descriptive Donachie model, as a third alternative into our code. 
In Fig. 4 the results of the three different Donachie models are compared. Values of the minimal median cell volume $(\mathrm{fl})$ were $0.51 \pm 0.01$ for the mechanistic model, $0.565 \pm 0.0004$ for the mechanistic model with eclipse period, and 0.396 \pm 0.0002 for the descriptive model. At growth rates $>1 \cdot 1 \mathrm{~h}^{-1}$, the simple mechanistic model results in unbalanced growth with periodically changing interdivision times (one period consists of the following sequence of interdivision times: long, short, shorter, short). The shift to unbalanced growth at higher growth rates was not due to $\mu$ approaching $\mu_{\max }$, but occurs above a particular threshold of $\mu$, as changing $\mu_{\max }$ showed. Reducing the delay time from $60 \mathrm{~min}$ to $30 \mathrm{~min}$ or below, well below the experimental value of $60 \mathrm{~min}$ (Table 2), prevented unbalanced growth. Both mechanistic models result in excessively large cell volumes. Unbalanced growth and oversized cells indicate an overshoot phenomenon which also occurs with linearly growing cells (data not shown). Only the descriptive model performs well. For this model, a relationship between the median cell volume predicted from the Donachie equation (6a) and the volume-at-division has to be used. Koch (1993) gives a median cell volume/birth volume ratio of 1.325 for perfect (no randomness) exponential growth, derived from the canonical size distribution. This is slightly lower than the value of 1.433 determined directly from our simulation results with non-random exponential growth, that is, not using a theoretical size distribution. We have used the ratio from our simulation while implementing the descriptive Donachie model. Note that the median cell volume can be regarded either as a measure averaged over the population or as a measure averaged over the cell cycle. It is used in the former sense as a constraint in population-based models and in the latter in our individual-based model, solely to convert cell volume parameters based on median volume into parameters based on volume-at-division.

It is apparent that a mechanistic cell division model needs to include positive and negative regulatory elements to give balanced growth. For our purposes, the descriptive Donachie model, though not mechanistic, is to be preferred since it simulates the dependence of cell size on growth rate correctly. Jaworska et al. (1996) used a formulation similar to our descriptive model. But they substituted $\mu$ for number of generations in $1 \mathrm{~h}$ in the Donachie equation (6a) and use a value of $0.8 \mathrm{fl}$ for the minimum reproductive volume, whereas the value of $0.4 \mathrm{fl}$ used here for $\bar{V}_{u}$ corresponds to a minimum reproductive volume of $0.558 \mathrm{fl}\left(2 \bar{V}_{u} / 1.433\right)$. Domach et al. (1984) used a detailed biochemical model for cell division successfully. In their mechanistic model, regulation of replication initiation is controlled by a negative feedback loop where the rate of synthesis of repressor and anti-repressor is simulated. Termination of replication then starts septum formation. This supports the view that negative feedback is necessary for a mechanistic cell division model. Their model requires detailed knowledge of synthesis rates of key proteins and is therefore not easily applicable to other bacteria.

\section{Growth synchrony}

Synchrony of growth has no causes; absence of synchrony does. The total lack of growth synchrony, though invariably observed in bacterial cultures, should not be taken for granted, as the division of a cell into perfectly equal daughter cells would give rise to a synchronous culture. Only if the offspring are not equal, and daughter cells grow with different rates and divide at different volumes, does asynchrony ensue. Since division can never be perfect, asynchrony is impossible to avoid completely in growing cultures. It improves the survival of the culture as a whole as compared to a hypothetical synchronous one because cells in different stages of the cell cycle are more vulnerable to particular hazards than cells in other stages.

Here, we simulate the loss of synchrony in order to test the quantitative importance of likely causes of desynchronization. How fast do variations in growth rate and volume-at-division desynchronize a culture? How strong is the effect of spatial heterogeneity on growth synchrony? Cultures synchronized by selection techniques, which are never perfectly synchronous at the beginning, lose their synchrony in about 3 generations (Ingraham et al., 1983). Considering the initial lack of synchrony, it might be estimated that a complete loss of synchrony takes about 5 generations. In our simulations, the degree of synchrony after 5 generations is about $25 \%$ with variation of $V_{\max }, 40 \%$ (that is already almost the minimum obtained) with variation of volume-atdivision, and $23 \%$ with variation of both (Fig. 5). Loss of synchrony appears to be slightly slower in our simulation using a reasonable estimate for the $\mathrm{CV}$ of these parameters ( $\mathrm{CV} 0 \cdot 1$ ). This might be due to other variations affecting synchrony additionally, such as unequal cell division.

To aid in summarizing and comparing synchrony data, a single-number measure of synchrony is essential. Several different measures for the degree of synchrony have been proposed (Blumenthal \& Zahler, 1962; Koppes et al., 1980; Cooper, 1991). However, having been used for growth in a constant and uniform environment, they are not general enough for our purposes. Also, established measures of synchrony have been criticized on the ground that they mix sharpness of cell number increase with the duration of that increase (Cooper, 1991). We propose a new measure that is derived entirely from analysis of the growth curve, thereby avoiding assumptions of a particular growth law, of constant growth rate, or of a well-mixed culture (Fig. 5). It can be regarded as describing the sharpness only of cell number increase. The difference of the $\log _{2}$ of the cell number between time-steps $n$ and $n+1, d l$, directly gives the degree of synchrony at time-step $n$ with a range of 0 (complete lack of synchrony) to 1 (complete synchrony). However, as $d l$ of course fluctuates immensely in the case of random variation of growth rate, cell volume at division, or spatially varying substrate concentration, it has to be treated as a statistic. We found the moving standard deviation of $d l, m S D(d l)$, to 

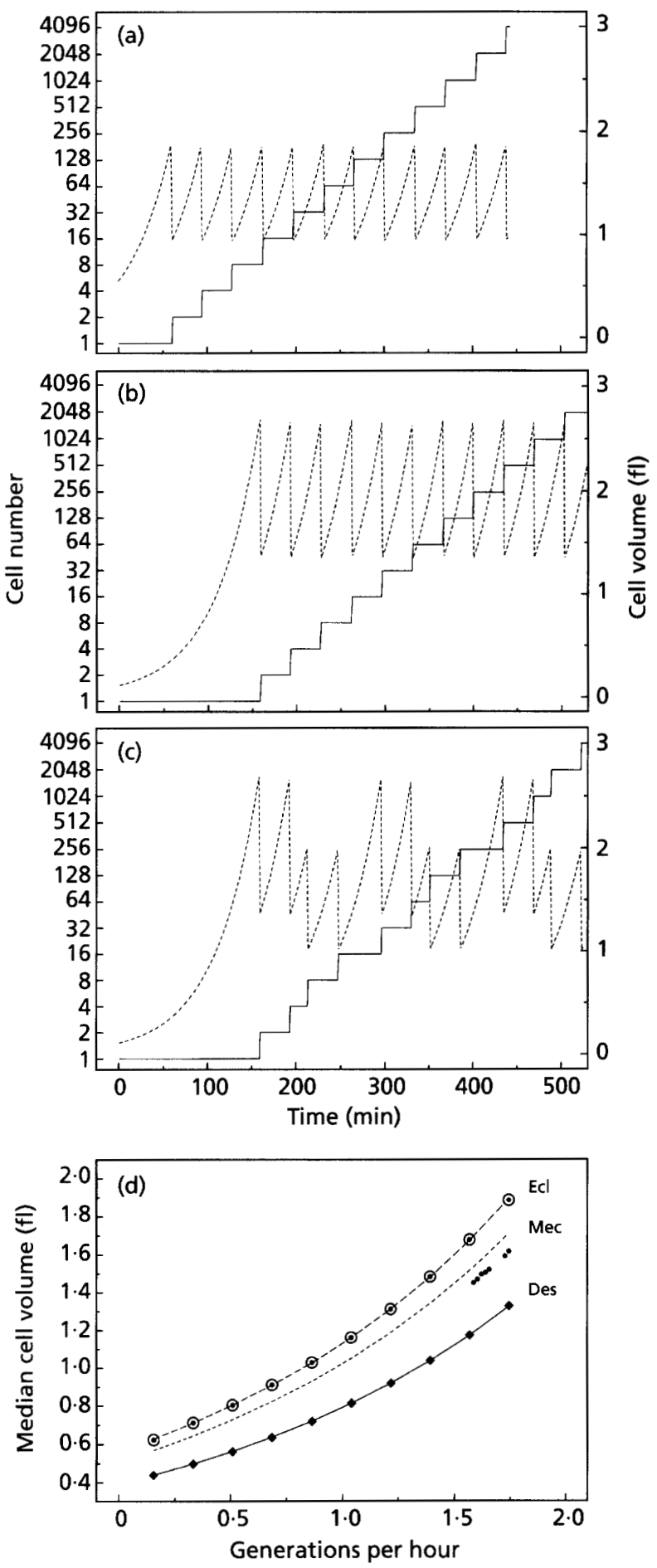

Fig. 4. $(a-c)$ Growth curves at the same and maximal growth rate for the three different implementations of the Donachie model: (a) descriptive, (b) mechanistic with eclipse, and (c) mechanistic without eclipse. - Cell number; --.., cell volume. Note that unbalanced growth as in the case of (c) occurs only at higher growth rates. (d) Cell cycle median cell volume as a function of generations per hour for the three different models. Symbols are data from individual simulations; lines represent non-linear fits of the data against the Donachie equation (6a) $\bar{V}=\bar{V}_{u} 2^{g}$, where $g$ is the number of generations in $1 \mathrm{~h}, \bar{V}$ is the median cell volume at a given $g$, and $\bar{V}_{u}$ is the minimal median cell volume. Des, descriptive model $(-, \bullet)$; be a suitable parameter. A window width equal to the minimum interdivision time was used. The window was moved in steps of one, starting with its centre at the time-step of the first division. $m S D(d l)$ can be normalized by dividing it by its theoretical maximum, which is a function of the number $n$ of data points (timesteps) contained in the window of width $w: m S D(d l)_{\max }$ $=\sqrt{1 / n} \cdot m S D(d l)$, after normalization, ranges from 0 to 1 , as does $d l$ itself. The intermediate values are well defined, as analysis of artificial mixtures of a synchronous and asynchronous simulated culture showed. $m S D(d l)$ is not affected by changes in population growth rate.

Random variation of cell parameters. In studying synchrony, parameters to vary randomly were chosen in order to have heterogeneity of cell size and growth rate in the culture. For the coefficient of variation $(\mathrm{CV})$ of the volume-at-division, a typical value of $0 \cdot 1$ (Koch \& Schaechter, 1962; Koch, 1993; Koch, 1996b) was used. (Available data give the $\mathrm{CV}$ of length-at-division. The $\mathrm{CV}$ for volume-at-division is almost as high as the $\mathrm{cV}$ for length-at-division if the variability of the width-atdivision is assumed to be small compared with lengthat-division.) We are not aware of quantitative data for the $\mathrm{CV}$ of $V_{\max }$ that are independent of the variation of size-at-division or which would allow a correction. Hence, we chose a value of $0 \cdot 1$. Jaworska et al. (1996) structured the population similarly by varying the maximal anabolic rate and minimum reproductive volume. Random variation of $V_{\max }$ led to complete desynchronization whereas random variation of the volume-at-division did not (Fig. 5). Variation of volumeat-division can be regarded as an imprecision of volume measurement by the cell and it broadens the size distribution and more so the age distribution. This view is substantiated since fluctuations in the size at one division are not correlated with the fluctuations in size at the previous division (Schaechter et al., 1962). Size variation does not affect growth rate if uptake and maintenance are proportional to biomass, as holds here. Even when size does not affect growth rate, a correct simulation of cell size and cell size variation is important since a large cell can survive longer without substrate and divide more readily upon resupply of substrate.

Spatial heterogeneity. Spatial heterogeneity of substrate concentration results from the balance of substrate uptake by the cells of the colony and the diffusion of substrate from the peripheral reservoir into the colony (Fig. 6).

Again, a single-number quantifier of spatial heterogeneity is essential for comparing results. As a very simple and entirely general new measure of spatial heterogeneity we propose the use of the standard

Ecl, mechanistic with eclipse $(---, 0)$; Mec, mechanistic without eclipse $(-\cdots, 0)$. Note that the latter two data sets are identical at lower growth rates but different at higher growth rates $\left(\mu\right.$ above $1.1 \mathrm{~h}^{-1}$, corresponding to 1.59 generations $\left.\mathrm{h}^{-1}\right)$. 

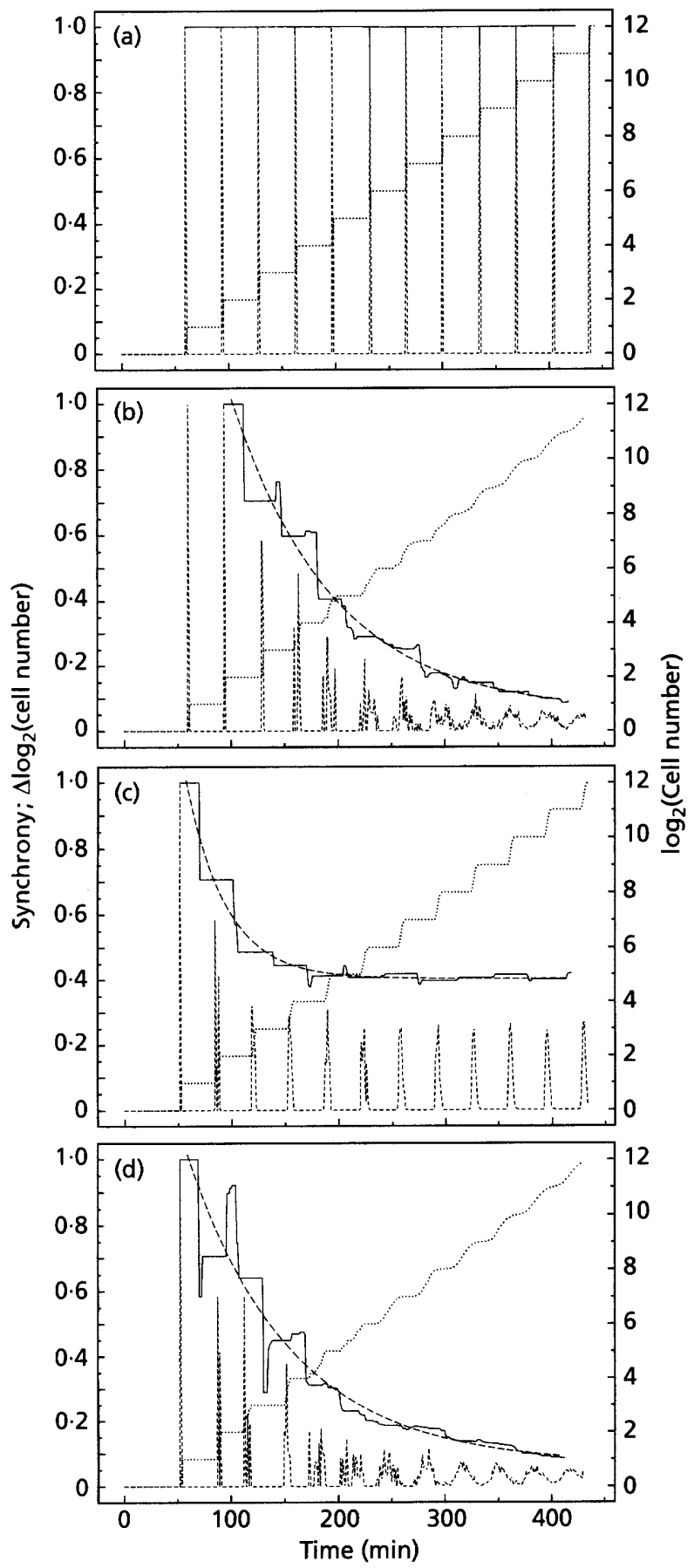

Fig. 5. Loss of growth synchrony due to random variation of cell parameters.,---- , Degree of synchrony $(-, m S D(d)$; --- , fit); $\cdots, \log _{2}$ (cell number); -.--, change of $\log _{2}$ (cell number) per time-step. An exponential function, $m S D(d l)=$ $1-a(1-\exp [-\tau(t-w / 4)])$, was fitted to the degree-of-synchrony trace $[m S D(d l)]$ with $(1-a)$ as the asymptote, $\tau$ as the time constant and $w$ as the window width. (a) Without random variation, no loss of synchrony. (b) Random variation of $V_{\text {max }}$ complete loss of synchrony (asymptote from fit is zero). (c) Random variation of volume-at-division, partial loss of synchrony. (d) Random variation of both parameters, complete loss of synchrony (asymptote from fit is zero). Loss of synchrony is exponential in all cases. deviation of substrate concentration in the areas occupied by cells. This can be computed in two ways. Either the occupied space can be divided into sections using the substrate concentration in each section, or the substrate concentration that is experienced by each cell is used for computing the SD (using $N$, the number of data points, as divisor since it is not a sample SD). The latter method weights the contribution of a particular area according to the number of cells it contains and is thus preferable. In both cases, the SD has two important properties. First, if the spatial resolution (grid granularity for example) is altered, the SD is not affected systematically. Second, if the size of the occupied space changes during colony development, or the number of cells occupying a particular zone changes, the SD is invariant. The $\mathrm{CV}$ can be used as a relative measure. A sharp increase in cell number, however, leads to temporary fluctuations due to the necessary reshuffling of cells following a burst of cell divisions (Fig. 6).

Spatial heterogeneity did not really desynchronize the cells since cells at the colony rim continued to grow synchronously with the rate the colony started off with. However, the population became increasingly subdivided into fractions growing at different rates, which resulted in apparent asynchrony.

\section{Conclusions}

Surface-area-related uptake and maintenance rates are appealing from a physico-chemical point of view but are not backed up experimentally. Simulations show the effect of surface dependence on cell growth to be minimal. For a population model of rod-shaped bacteria, it appears appropriate to disregard cell shape and surface area.

Of several simple implementations of a cell division model, only the descriptive version of the Donachie model correctly reproduced the cell size dependence on growth rate.

Growth synchrony is not completely lost if the cell measures volume-at-division imprecisely (no hereditary changes), but a $\mathrm{CV}$ of 0.1 for $V_{\max }$ is largely sufficient to explain the rate of loss observed in cultures synchronized by selection techniques. Desynchronization follows exponential decay kinetics in both cases of random variation of cell parameters but not if it is due to increasing spatial heterogeneity.

In two cases, it was necessary to formulate new measures to describe simulation results. With growth synchrony, the moving SD of the derivative of the logarithm of the growth curve $[m S D(d l)]$, and with spatial heterogeneity, the $\mathrm{CV}$ of substrate concentration or whatever is to be considered, were found to be useful universal measures. Both quantities can be measured. For $m S D(d l)$, the growth curve of the population is needed. For the CV of substrate concentration or growth rate, measurements with microelectrodes or modern microscopic techniques are needed. 

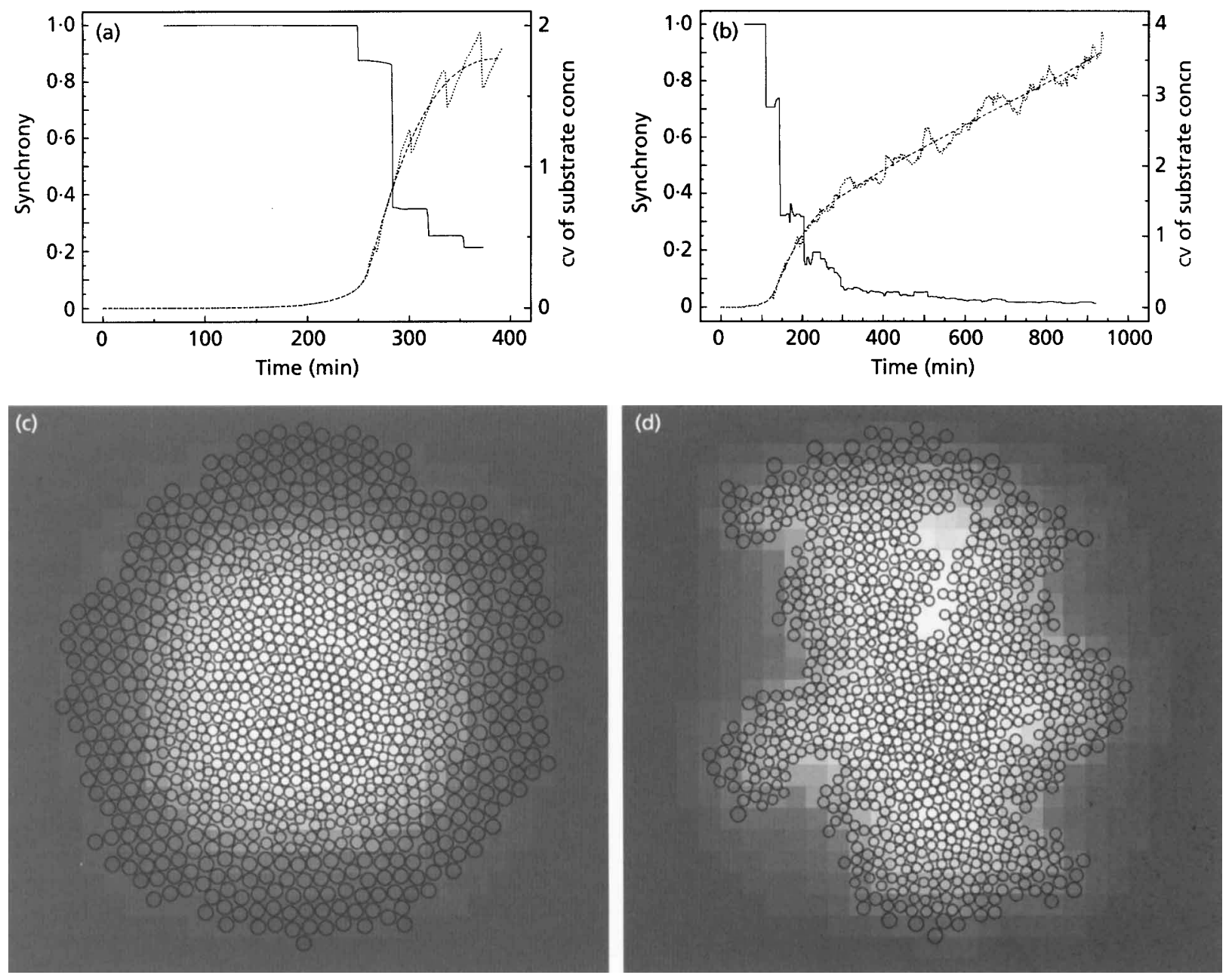

Fig. 6. (a, b). Loss of growth synchrony due to increasing spatial heterogeneity (no random variation of cell properties). Glucose concentration was $1 \mathrm{fg} \mathrm{fl}^{-1}$ (a) and $0.1 \mathrm{fg} \mathrm{fl}^{-1}$ (b). _-, Degree of synchrony $[m S D(d l)] ; \ldots, \ldots$, spatial heterogeneity (..., CV of substrate concentration; -.-., spline fit). Note the fluctuations due to synchronous growth; bursts of divisions leading to rearrangements of cellular positions cause temporary fluctuations of the $\mathrm{CV}$ as a measure of spatial heterogeneity (if calculated by looping through all cells' exterior substrate concentration). Loss of synchrony typically does not follow a simple exponential decay function. (c, d) Screen shots. Spatial heterogeneity at the end of runs (about 1000 cells) at reservoir substrate concentrations of $1 \mathrm{fg} \mathrm{fl}^{-1}$ (c), $391 \mathrm{~min}$, and $0.1 \mathrm{fg} \mathrm{fl}^{-1}$ (d), $939 \mathrm{~min}$. At $10 \mathrm{fg} \mathrm{fl}^{-1}$, the spatial heterogeneity stays very low $(\mathrm{cv}<0.1)$ and is not visible on screen shots; also, growth synchrony stays perfect (data not shown). The substrate concentration gradient is visualized as follows: the darker the square, the higher the concentration (logarithmic scale). The substrate concentration in the middle of panels (c) and (d) is too low to support significant uptake rates. Cells in the middle shrink at a maximal rate which is equal to the maintenance rate. The grid size used, of $2 \times 2 \mu \mathrm{m}$, was sufficiently small. Simulations including random variation of cell parameters look very similar (data not shown).

During this study, the lack of information on the individual heterogeneity of growth parameters became increasingly apparent to us. Most reports on this subject are part of the early work on cell division and focused on size-at-division variability. We feel that a revival of research in this area employing modern techniques would be rewarding. Another area where information required for modelling is insufficient is the volume fraction occupied by cells in colonies and biofilms. The most appropriate study in this regard is a statistical analysis of cellular volume fractions in biofilms, although cell cluster areas were not treated separately (Korber et al., 1993). We are not aware of a similar study for colonies. Sadly, this so far precludes a quantitative comparison of our simulated colony shapes and structures with real colonies.

Qualitatively, the simulated colony structure at low glucose concentration $\left(0 \cdot 1 \mathrm{~g} \mathrm{l}^{-1}\right)$ resembles the fractal colony patterns observed experimentally and with diffusion-limited aggregation (DLA) models (Matsushita, 1997; Wimpenny \& Colasanti, 1997). These patterns emerge in simulations where particles diffuse randomly starting at the periphery (slow process) and become stuck when hitting a cluster (fast process). In our simulation, the mechanisms are somewhat 
different; metaphorically speaking, food particles stick to agents but agents themselves are not sticky, have variable extent, and grow not only at the cluster surface. Nevertheless, the DLA pattern results.

BacSim shows that using readily available parameters, it is possible to construct a spatially explicit IbM of bacterial population growth that is quantitatively correct. The model will be extended to allow simulation of growth at a larger spatial scale as well as cell differentiation and pheromone signalling, aiming at modelling multi-species biofilms. Due to the flexibility and generality of the object-oriented model structure, we expect considerable leverage in building new applications.

IbM of bacteria has great promise. The task of understanding a given complex adaptive system requires integration of analytical and synthetic approaches. It is not finished after an experimental dissection of the system into its parts and the study of these parts, as the question remains whether we can reconstruct the behaviour of the complete, macroscopic system by putting what we have learnt about these pieces back together again in a model. IbM is ideal for this as it does not suffer from limitations in principle. We hope to have convinced our readers that IbM is also feasible for bacteria.

\section{ACKNOWLEDGEMENTS}

We gratefully acknowledge helpful discussions about modelling with Dr Gary Barker, Institute for Food Research, Norwich, UK, and about statistical analysis with Professor A. Zhigliavsky, Cardiff University. The timely and expert help from the Swarm user community on design and coding questions is very much appreciated. J.-U.K. wishes to thank the DFG (Deutsche Forschungsgemeinschaft, grant Kr 1616/21) and G.B. the NSF (grant no. BIR 9400642) for financial support.

\section{REFERENCES}

Adam, G., Läuger, P. \& Stark, G. (1977). Physikalische Chemie und Biophysik. Berlin: Springer.

Ben-Jacob, E., Schochet, O., Tenenbaum, A., Cohen, I., Czirok, A. \& Vicsek, T. (1994). Generic modeling of cooperative growthpatterns in bacterial colonies. Nature 368, 46-49.

Blumenthal, L. K. \& Zahler, S. A. (1962). Index for measurement of synchronization of cell populations. Science 135, 724 .

Booth, G. (1997). Gecko: a continuous 2-D world for ecological modeling. Artif Life 3, 147-163.

Button, D. K. (1993). Nutrient-limited microbial growth kinetics: overview and recent advances. Antonie Leeuwenboek 63, 225-235.

Cooper, S. (1991). Bacterial Growth and Division. San Diego: Academic Press.

Davey, H. M. \& Kell, D. B. (1996). Flow cytometry and cell sorting of heterogeneous microbial populations: the importance of singlecell analyses. Microbiol Rev 60, 641-696.

DeAngelis, D. L. \& Gross, L. J. (1992). Individual-based Models and Approaches in Ecology: Populations, Communities, and Ecosystems. New York: Chapman \& Hall.
Domach, M. M., Leung, S. K., Cahn, R. E., Cocks, G. G. \& Shuler, M. L. (1984). Computer model for glucose-limited growth of a single cell of Escherichia coli B/r-A. Biotechnol Bioeng 26, 203-216.

Donachie, W. D. (1968). Relationship between cell size and time of initiation of DNA replication. Nature 219, 1077-1079.

Donachie, W. D. \& Robinson, A. C. (1996). Cell division: parameter values and the process. In Escherichia coli and Salmonella typhimurium: Cellular and Molecular Biology, 2nd edn, pp. 1578-1593. Edited by F. C. Neidhardt and others. Washington, DC: American Society for Microbiology.

Grimson, M. J. \& Barker, G. C. (1994). Continuum model for the spatiotemporal growth of bacterial colonies. Phys Rev E 49, 1680-1684.

Helmstetter, C. E. (1996). Timing of synthetic activities in the cell cycle. In Escherichia coli and Salmonella typhimurium: Cellular and Molecular Biology, 2nd edn, pp. 1627-1639. Edited by F. C. Neidhardt and others. Washington, DC: American Society for Microbiology.

Herbert, D. (1958). Some principles of continuous culture. In Resumés de Travaux Presentées à Sessions de Rapports: 7 th Congrès International de Microbiologie, Stockholm, pp. 381-396. Edited by G. Tunevall. Uppsala: Almqvist \& Wiksells.

Hughes, W. H. (1955). The inheritance of differences in growth rate in Escherichia coli. J Gen Microbiol 12, 265-268.

Ingraham, J. L., Maaløe, O. \& Neidhardt, F. C. (1983). Growth of the Bacterial Cell. Sunderland, MA: Sinauer Associates.

Jaworska, J. S., Hallam, T. G. \& Schultz, T. W. (1996). A community model of ciliate Tetrabymena and bacteria E. coli. Part 1 . Individual-based models of Tetrahymena and E. coli populations. Bull Math Biol 58, 247-264.

Jeong, J. W., Snay, J. \& Ataai, M. M. (1990). A mathematical model for examining growth and sporulation processes of Bacillus subtilis. Biotechnol Bioeng 35, 160-184.

Joshi, A. \& Palsson, B. O. (1988). Escherichia coli growth dynamics: a three-pool biochemically based description. Biotechnol Bioeng 31, 102-116.

Koch, A. L. (1985). The macroeconomics of bacterial growth. In Bacteria in Their Natural Environments, pp. 1-42. Edited by $M$. Fletcher \& G. D. Floodgate. London: Academic Press.

Koch, A. L. (1993). Biomass growth rate during the prokaryote cell cycle. Crit Rev Microbiol 19, 17-42.

Koch, A. L. (1996a). What size should a bacterium be? A question of scale. Annu Rev Microbiol 50, 317-348.

Koch, A. L. (1996b). Similarities and differences of individual bacteria within a clone. In Escherichia coli and Salmonella typhimurium: Cellular and Molecular Biology, 2nd edn, pp. 1640-1651. Edited by F. C. Neidhardt and others. Washington, DC: American Society for Microbiology.

Koch, A. L. (1997). Microbial physiology and ecology of slow growth. Microbiol Mol Biol Rev 61, 305-318.

Koch, A. L. \& Schaechter, M. (1962). A model for statistics of the cell division process. J Gen Microbiol 29, 435-454.

Koch, A. L. \& Wang, C. H. (1982). How close to the theoretical diffusion limit do bacterial uptake systems function? Arch Microbiol 131, 36-42.

Kooijman, S. A. L. M., Muller, E. B. \& Stouthamer, A. H. (1991). Microbial growth dynamics on the basis of individual budgets. Antonie Leeuwenhoek 60, 159-174.

Koppes, L. J. H., Meyer, M., Oonk, H. B., de Jong, M. A. \& Nanninga, N. (1980). Correlation between size and age at different 
events in the cell division cycle of Escherichia coli. J Bacteriol 143, 1241-1252.

Korber, D. R., Lawrence, J. R., Hendry, M. J. \& Caldwell, D. E. (1993). Analysis of spatial variability within $\mathrm{mot}^{+}$and $\mathrm{mot}^{-}$ Pseudomonas fluorescens biofilms using representative elements. Biofouling 7, 339-358.

Matsushita, M. (1997). Formation of colony patterns by a bacterial population. In Bacteria as Multicellular Organisms, pp. 366-393. Edited by J. A. Shapiro \& M. Dworkin. New York: Oxford University Press.

Minar, N., Burkhart, R., Langton, C. \& Askenazi, M. (1996). The Swarm simulation system: a toolkit for building multi-agent simulations. SFI Working Paper 96-06-042 (http:// www.santafe.edu/sfi/publications).

Neidhardt, F. C. \& Umbarger, H. E. (1996). Chemical composition of Escherichia coli. In Escherichia coli and Salmonella typhimurium: Cellular and Molecular Biology, 2nd edn, pp. 13-16. Edited by F. C. Neidhardt and others. Washington, DC: American Society for Microbiology.

Neijssel, O. M., de Mattos, M. J. T. \& Tempest, D. W. (1996). Growth yield and energy distribution. In Escherichia coli and Salmonella typhimurium: Cellular and Molecular Biology, 2nd edn, pp. 1683-1692. Edited by F. C. Neidhardt and others. Washington, DC: American Society for Microbiology.

Picioreanu, C., van Loosdrecht, M. C. M. \& Heijnen, J. J. (1998a). A new combined differential-discrete cellular automaton approach for biofilm modeling: application for growth in gel beads. Biotechnol Bioeng 57, 718-731.

Picioreanu, C., van Loosdrecht, M. C. M. \& Heijnen, J. J. (1998b). Mathematical modeling of biofilm structure with a hybrid differential-discrete cellular automaton approach. Biotechnol Bioeng 58, 101-116.

Pirt, S. J. (1965). The maintenance energy of bacteria in growing cultures. Proc R Soc London B 163, 224-231.

Pirt, S. J. (1967). A kinetic study of the mode of growth of surface colonies of bacteria and fungi. J Gen Microbiol 47, 181-197.

Schaechter, M., Williamson, J. P., Hood, J. R., Jr \& Koch, A. L. (1962). Growth, cell and nuclear divisions in some bacteria. J Gen Microbiol 29, 421-434.

Schmitz, O. J. \& Booth, G. (1997). Modeling food web complexity: the consequence of individual-based spatially explicit behavioral ecology on trophic interactions. Evol Ecol 11, 379-398.

Shapiro, J. A. \& Dworkin, M. (1997). Bacteria as Multicellular Organisms. New York: Oxford University Press.

Shuler, M. L., Leung, S. K. \& Dick, C. C. (1979). A mathematical model for the growth of a single bacterial cell. Ann N Y Acad Sci 326, 35-55.

Tempest, D. W. \& Neijssel, O. M. (1984). The status of $Y_{\text {ATP }}$ and maintenance energy as biologically interpretable phenomena. Annu Rev Microbiol 38, 459-486.

Wimpenny, J. W. T. (1992). Microbial systems: patterns in time and space. Adv Microb Ecol 12, 469-522.

Wimpenny, J. W. T. \& Colasanti, R. (1997). A unifying hypothesis for the structure of microbial biofilms based on cellular automaton models. FEMS Microbiol Ecol 22, 1-16.

Received 24 April 1998; revised 11 August 1998; accepted 17 August 1998. 